\title{
Conciencia y trabajo continuo como predictores del rendimiento académico en estudiantes españoles ${ }^{1}$
}

\section{Conscientiousness and laboriousness as academic achievement predictors in a Spanish students sample}

\author{
Teresa DEZCALLAR SÁEZ ${ }^{1}$, Mercè CLARIANA MUNTADA ${ }^{1}$, \\ María del Mar BADIA MARTÍN ${ }^{1}$, Concepció GOTZENS BUSQUETS ${ }^{2}$ \\ y Ramón CLADELLAS PROS ${ }^{1}$ \\ ${ }^{1}$ Universidad Autónoma de Barcelona y ${ }^{2}$ Universidad Illes Balears
}

Recibido: Octubre 2013

Aceptado: Abril 2014

\begin{abstract}
Resumen
La conciencia como factor de personalidad del modelo Big Five es una variable clave para entender los resultados académicos de los alumnos. El objetivo de este artículo es analizar la relación que establecen la propia conciencia y dos de sus facetas, el trabajo continuo y la planificación, con el rendimiento académico, y observar si hay diferencias con la variable género. Un total de 456 alumnos españoles de bachillerato y universidad han participado en el estudio. Se han administrado unos cuestionarios y un autoinforme para la obtención de los datos del estudio. Los resultados demuestran que tanto la conciencia como dimensión de personalidad como la faceta trabajo continuo son capaces de predecir aspectos esenciales del rendimiento, concretamente, las notas de los alumnos en los exámenes, su asistencia a clase y su esfuerzo o dedicación a los estudios. La variable género muestra una diferencia a nivel de factor conciencia, siendo más elevada en el género femenino. Desde una perspectiva práctica, estos resultados indican que incluir, entre los objetivos educativos, la instauración de un hábito de trabajo continuado, es adecuado para mejorar tanto las notas de los alumnos como su adaptación al ámbito educativo.
\end{abstract}

Palabras clave: conciencia, big five, trabajo continuo, planificación, examen, asistencia, dedicación, estudio.

\begin{abstract}
To understand academic performance of students, the variable of conscientiousness from personality inventory Big Five, has been recognized as an important key. The aim of this paper is to analyze the relationship established between the personality factor conscientiousness itself and two of its facets, laboriousness and planning, with academic performance, and observe if
\end{abstract}

\footnotetext{
${ }^{1}$ Este estudio se ha llevado a cabo dentro del proyecto EDU 2009-10651 del Ministerio de Ciencia e Innovación de España. 
there are genre differences in consciousness personality factor. A total of 456 Spanish students of high school and college participated in the study. They were requested to answer a personality report and a self inform questionnaire. The results show that both conscientiousness as a personality dimension and the consideration of laboriousness facet are able to predict academic performance, especially with regard to student's exam marks, classroom attendance and dedication to study. The genre variable pointed out that feminine genre is more conscious than male in that personality factor. From a practical perspective, these results indicate that the establishment of a routine of continuous work is suitable for improving student grades and their adaptation to the educational environment.

Keywords: conscientiousness, big five, laboriousness, task planning, exam marks, class attendance, study dedication.

Existe evidencia empírica suficiente para afirmar que la personalidad, de manera similar a la inteligencia, es también relevante a la hora de explicar los resultados académicos (Laidra, Pullman y Allik, 2007). De este modo, ambos aspectos, lo que la persona puede hacer -que sería la inteligencia- y el cómo la persona lo hace -que correspondería a la personalidad- resultan ser predictores significativos en el mundo académico (Chamorro-Premuzic y Furham, 2003). Esta concepción desmiente en parte la que defendían algunos estudios de finales de la década de los noventa, que afirmaban que la inteligencia predecía el aprovechamiento académico mejor que la personalidad (por ejemplo Sternberg y Kaufman, 1998), o que la personalidad no era un factor determinante en los contextos educacionales (Allik y Realo, 1997).

Actualmente, se acepta de forma relativamente unánime que la personalidad es un aspecto notable para comprender el funcionamiento de una persona en su entorno educativo. En la revisión de Fullana (1993) sobre las variables que explican el rendimiento académico, se consideraba la personalidad, aptitudes y actitudes como elementos importantes. Los pedagogos añadían a sus variables acerca del currículo, los conocimientos y las destrezas cognitivas, variables como el nivel de organización personal, el temperamento, la afectividad: variables todas de tipo emocional y de personalidad que pueden condicionar el rendimiento académico y que estarán vinculadas a otros factores explicativos. En este marco, la teoría de las diferencias individuales entiende los factores de personalidad como algo sustancial y esencial de cada persona, y no sólo como algo residual al servicio de la inteligencia. En relación a este punto, se ha comprobado que los estudiantes difieren en su dedicación al estudio y en el mantenimiento de la atención en clase, en base a las diferencias de contexto y de personalidad (Stipek, 2002), así como en su actitud frente a la educación (Conard, 2006) y en el uso de determinadas estrategias de aprendizaje (Duff, Boyle, Dunleavy y Ferguson, 2004).

\section{Conciencia y aprovechamiento académico}

Hasta la fecha, diversos autores han remarcado la relación entre conciencia y rendimiento académico. Por ejemplo Digman afirma que "Se encuentra asociación consistente entre conciencia (voluntad de logro), desempeño laboral y logro 
académico." (Digman, en Guzmán, Calderón y Cassaretto, 2003, p.122). La relación existente entre valores elevados del factor conciencia y el aprovechamiento académico es la voluntad de cumplir tareas, la convicción de obtener metas altas y la puesta en marcha de habilidades de organización y trabajo duro, así como de la solución de problemas.

Para examinar las relaciones existentes entre el rendimiento académico y la personalidad se han utilizado principalmente los modelos de los cinco grandes factores, como el FFM (Five-Factor Model; O'Connor y Paunonen, 2007) o el BFI (Big Five Inventory; Costa y McCrae, 1992). Como es sabido, estas propuestas describen cinco factores o dimensiones de la personalidad: Amabilidad (A), Conciencia (C), Extraversión (E), Neuroticismo (N), y Apertura a la experiencia (O). Estos factores han demostrado su validez en estudios psicológicos (Noftle y Robins, 2007; O'Connor y Paunonen, 2007) y, más concretamente, en numerosas ocasiones, el factor $\mathrm{C}$ ha resultado correlacionar alta y positivamente con el rendimiento académico (Beaujean, Firmin, Attai, Johnson, Firmin y Mena2011; Duff, Boyle, Dunleavy y Ferguson, 2004; Johnson y Bloom, 1995; Komarraju, Karau, Schmeck y Avdic, 2011; MacCann, Duckworth y Roberts, 2009; Poropat, 2009), y también con la motivación y la persistencia en el logro de buenos resultados en la escuela (Chamorro-Premuzic y Furnham, 2003; Komarraju y Karau, 2005; Poropat, 2009; Ziegler, Knogler y Bühner, 2009).

Además de estudiar la incidencia de la conciencia en el aprendizaje académico, algunos autores también han analizado la influencia que tiene cada una de sus facetas del inglés facet- en los resultados educativos. En publicaciones recientes, MacCann y colaboradores (MacCann et al., 2009) identifican diferentes modelos y medidas de las facetas de la C (además del FFM y el BFI, ya citados; el NEO/BPQ de Perugini y Gallucci, 1997; los adjetivos ABF5C de Saucier y Ostendorf, 1999; o el AB5C de Peabody y De Raad, 2002; entre otros). Entre las similitudes halladas en los cuestionarios estudiados, estos autores destacan que son ocho las facetas de la conciencia más comúnmente aceptadas: el trabajo continuo, el perfeccionismo, el orden, el autocontrol, la precaución, la planificación del trabajo, la perseverancia, y la puntualidad o falta de procrastinación. Estas ocho facetas han mostrado de forma consistente correlaciones significativas, positivas y altas con el rendimiento académico (por ejemplo: Busato, Prins, Elshout y Hamaker, 2000; Costa y McCrae, 1992; De Raad y Schouwenburg, 1996). A nivel más particular, las facetas de la conciencia que han demostrado establecer una asociación más estrecha con el rendimiento académico son la planificación y el trabajo continuo (Lounsbury, Sundstrom, Loveland y Gibson, 2003).

Precisamente estos dos aspectos de la conciencia como dimensión de personalidad, el trabajo continuado -del inglés industriousness- y la planificación -traducido de task planning-, son las dos facetas de la $\mathrm{C}$ que se han escogido en el presente estudio, para analizar su relación con el rendimiento académico. El rendimiento académico, se mide habitualmente a través de las notas de exámenes correspondientes al alumno en cuestión a lo largo de un semestre o de un curso, así como con indicadores de absentismo, conductas a favor o en contra de la convivencia en el aula o asistencia y 
participación en clase, generalmente en forma de informes por parte de profesores, padres, tutores o del propio alumno (de la Fuente, Pichardo, Justicia y Berbén, 2008).

Asimismo, el factor conciencia, por su parte, se asocia al éxito académico tomando como unidad de análisis, mayoritariamente, las notas de los exámenes (ChamorroPremuzic y Furham, 2003; Noftle y Robins, 2007; Wagerman y Funder, 2007), y también se correlaciona con la capacidad de atención plena durante el proceso de enseñanza y aprendizaje (León, 2008). Por otro lado, la asistencia al aula, como variable controlable por la propia persona, y la dedicación al estudio, también han hecho patente su relación con el factor conciencia (Dollinger, Matyja y Huber, 2008) y han demostrado ser de interés para la consecución del éxito académico.

Cabe añadir, que en general, la asistencia a clase, o en su vertiente opuesta, el absentismo escolar, ha sido una variable poco estudiada en relación a factores de personalidad. Sin embargo, los pocos estudios realizados hasta la fecha apuntan a una correlación positiva y significativa entre el número de ausencias en clase y la procrastinación (Clariana, 2008) o el bajo rendimiento académico (Rose, Hall, Bolen y Webster, 1996). Por otra parte, algunos autores (Schuman, Walsch, Olson y Etheridge, 1985) han observado que la asistencia a clase tiene un efecto menor en el rendimiento académico en comparación con la dedicación al estudio o las capacidades cognitivas. No queremos dejar de mencionar la existencia de algunos expertos (Conard, 1996) que han informado de que la asistencia a clase forma parte de un clúster de variables válidas para predecir el buen rendimiento en el ámbito académico, junto con la conciencia como dimensión de personalidad y la dedicación al estudio. Asimismo, la asistencia a clase junto con la motivación en estudiantes universitarios, según Pérez, Ramón y Sánchez (en Garbanzo, 2007), se asocia a un mejor rendimiento académico.

En cuanto a las diferencias de género, son escasos los estudios que tienen en cuenta este factor a la hora de predecir el rendimiento académico en base a los factores de personalidad. Cabe citar, sin embargo, a autores como Carrasco y Del Barrio (2007), Del Barrio, Del Barrio y Holgado (2006), y Niño de Guzmán, Calderón y Cassaretto (2003), hallaron que el género femenino se caracterizaba por obtener habitualmente puntuaciones más altas en la dimensión de conciencia. No obstante, otros estudios no han hallado diferencias significativas entre ambos géneros (Weisberg, DeYoung y Hirsh, 2011).

Como señala Garbanzo (2007), las investigaciones acerca del rendimiento académico van asociadas a una búsqueda de calidad en la educación y en un planteamiento integral que analice y tenga en cuenta la interacción de factores personales, sociales e institucionales, los cuales son imprescindibles a la hora de tomar decisiones dentro del sistema educativo. Así que podríamos entrever el interés de algunos de estos factores, como el de la variable personalidad, entre otros, como una aproximación a la realidad educativa. 


\section{Objetivos de la investigación}

El objetivo de este trabajo es analizar la capacidad de dos facetas de la conciencia como dimensión de personalidad, el trabajo continuo y la planificación por separado, así como la variable factor $\mathrm{C}$ en su globalidad, para predecir el rendimiento académico de estudiantes a partir de la educación no obligatoria, en concreto, bachillerato y universidad.

Además, se estudiará si se encuentran diferencias en cuanto a género en relación con la conciencia y el rendimiento académico.

\section{Método}

\section{Participantes}

La muestra del estudio está compuesta por 456 estudiantes españoles de bachillerato y universidad que participaron de forma voluntaria. Se seleccionaron tres centros de Barcelona y dos de Madrid, tanto privados como públicos y con una representatividad heterogénea de variables sociológicas. Los centros fueron seleccionados mediante muestreo no aleatorio incidental, por el único motivo de viabilidad de acceso. La edad de los participantes está comprendida entre los 16 y los 30 años de edad, siendo la media de $18,81(\mathrm{SD}=2,85)$ años. Del total de la muestra, 165 alumnos son varones (36,18\%), 291 son mujeres $(63,82 \%), 284(62,28 \%)$ estaban cursando bachillerato en el momento del estudio y $172(37.72 \%)$ hacían la carrera de Psicología. El género se reparte de forma equilibrada entre los alumnos de bachillerato (50,70\% y 49,30\%) mientras que en la carrera de Psicología, tal como suele ocurrir habitualmente, las mujeres están más representadas $(87,79 \%)$. Se excluyeron de la muestra los alumnos menores de 16 años debido a la utilización de inventarios de personalidad que requerían ser mayor de esa edad, así como aquellos alumnos que tuvieran algún tipo de necesidad educativa específica.

\section{Instrumentos y variables}

Los alumnos contestaron un cuestionario anónimo autodescriptivo que preguntaba sobre el nivel de estudios, el género y la edad. Además, incluía la versión $\mathrm{S}$ o reducida del Big Five Inventory de Rammstedt y John (BFI10; 2007) y dos de las facetas de la C conciencia propuestas por McCann y colaboradores (McCann et al., 2009): la denominada Conscientiousness Task Planning ("Cplan" a partir de ahora, con 9 ítems) y la de Conscientiousness Industriousness (que denominaremos "Cind" a continuación, y que tiene 10 ítems). Tanto las dimensiones de personalidad del Big Five como estas dos facetas de la $\mathrm{C}$ tenían cinco opciones de respuesta en una escala tipo Likert de cinco puntos, desde " 1 = Absolutamente en desacuerdo" hasta " $5=$ Absolutamente de acuerdo". Asimismo, todas las escalas contenían ítems que puntuaban en sentido inverso. 
Para más claridad se añade a continuación en la Tabla 1 la traducción que se ha utilizado de las dos facetas de la conciencia:

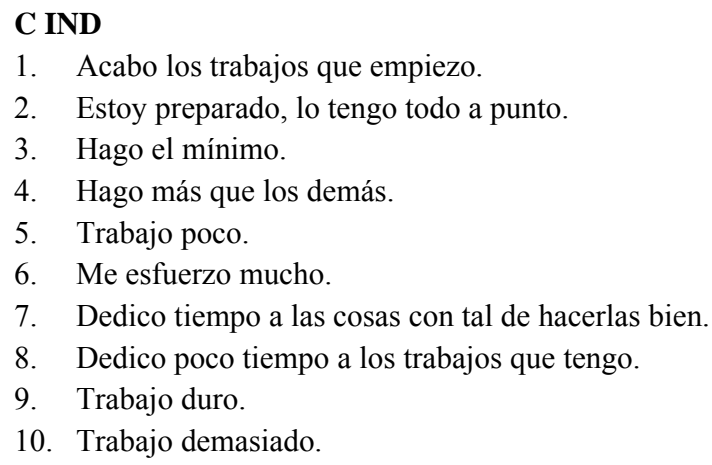

1. Acabo los trabajos que empiezo.

2. Estoy preparado, lo tengo todo a punto.

3. Hago el mínimo.

4. Hago más que los demás.

5. Trabajo poco.

6. Me esfuerzo mucho.

7. Dedico tiempo a las cosas con tal de hacerlas bien.

8. Dedico poco tiempo a los trabajos que tengo.

9. Trabajo duro.

10. Trabajo demasiado.

\section{PLAN}

1. Trabajo para conseguir mis objetivos.

2. Hago las cosas según un plan.

3. Sigo mis planes.

4. Me gusta seguir las normas.

5. Me gusta planificar el trabajo con antelación.

6. Hago planes y luego los sigo.

7. Si tomo una decisión después no la cambio.

8. Sigo mis propósitos.

9. Tengo hábitos de estudio y los sigo.

Tabla 1. Ítems de las facetas de Trabajo continuo y Planificación.

En base a los trabajos anteriores, en el presente estudio decidimos tomar tres variables como indicadoras del rendimiento académico del alumno: las calificaciones a través de exámenes, que de ahora en adelante denominaremos notas, el porcentaje de asistencia a clase, que denominaremos asistencia, y finalmente el porcentaje de su tiempo libre que el alumno dedica al estudio, al que nos referiremos como dedicación al estudio.

Para valorar el aprovechamiento académico, se pidió a los estudiantes que redactaran un informe sobre ellos mismos como alumnos, en el que se recogía información sobre la media de notas académicas del último semestre, la valoración de la asistencia a clase, considerada ésta como el porcentaje de asistencia siendo la completa asistencia, sin ningún absentismo el $100 \%$, y el porcentaje estimado de dedicación al estudio, considerado como el tiempo contemplado de horas de estudio fuera del horario académico del aula.

En resumen, las variables que se han utilizado en el presente trabajo son las que se detallan a continuación:

- Género. Variable independiente cualitativa de dos categorías, hombre y mujer.

- Dimensiones de personalidad, variables independientes continuas medidas cada una en una escala de 2 a 10 puntos: 
$>$ E extraversión.

$>$ A amabilidad.

$>$ C conciencia.

$>\mathrm{N}$ estabilidad emocional (cabe notar que esta escala se ha utilizado en sentido inverso a como se utiliza habitualmente, de modo que las puntuaciones altas indican mayor estabilidad emocional).

$>\mathrm{O}$ apertura mental.

- Facetas de la C, variables independientes continuas:

$>$ Cplan, planificación, escala con un rango de 9 a 45 puntos.

$>$ Cind, trabajo persistente y continuado, escala de 10 a 50 puntos.

- Indicadores de aprovechamiento académico, variables dependientes contínuas:

Notas. Media de notas del último semestre de acuerdo a la escala habitual de 0 -nota mínima- a 10 puntos -nota máxima o más alta-.

Assis, asistencia a clase. Valorada en porcentajes en una escala de 0 -no asiste nunca- a 100 puntos -siempre va a clase-.

$>$ Ded, dedicación al estudio, también valorada en porcentajes en una escala de 0 -no se dedica a las tareas académicas- a 100 -dedica todo su tiempo libre a realizar actividades relacionadas con el estudio-.

\section{Procedimiento}

En un inicio se contactó con centros educativos de bachillerato para administrar los cuestionarios y el informe a los alumnos. Ambos documentos se consensuaron previamente con los directores pedagógicos de los centros, dando un consentimiento informado, y su administración la realizaron los propios investigadores en una hora de tutoría.

Asimismo, se administró el material a alumnos de tercer curso del grado de Psicología de la Universitat Autònoma de Barcelona, UAB (España), un centro público con una gran cantidad de alumnos. Los estudiantes contestaron el cuestionario y cumplimentaron el informe durante la hora de clase de Psicología de la educación.

Una vez entregados los cuestionarios y los informes los alumnos de la muestra recibieron posteriormente retroalimentación de los resultados en forma de clase conjunta, en la que se explicó el objetivo del trabajo y las variables que se evaluaban, agradeciéndoles asimismo su participación.

\section{Análisis de los datos}

Una vez recopilados los datos se procedió a su análisis mediante el paquete estadístico SPSS 15.0 para Windows. 
A continuación, mediante una prueba t de Student se analizaron las diferencias entre géneros en las variables continuas del trabajo (factores de personalidad, notas académicas, asistencia a clase y dedicación al estudio). Puesto que la mayoría fueron significativas las últimas pruebas estadísticas de la investigación se llevaron a cabo separadamente para hombres y mujeres.

Para finalizar se calcularon ANOVA por géneros para comprobar la influencia de las variables de personalidad $\mathrm{C}, \mathrm{C}$ planificación y $\mathrm{C}$ trabajo continuo sobre los indicadores de adaptación académica, las notas, la asistencia a clase y la dedicación al estudio. Para ello, se convirtieron las variables de la conciencia de continuas a discontinuas de tres categorías, en función del percentil 25,50 o 75 en el que cada puntuación se situaba.

\section{Resultados}

En primer lugar se estudió la correlación de Pearson entre variables de personalidad e indicadores de aprovechamiento académico, lo que constató que la dimensión de personalidad que presenta una relación más estrecha con el rendimiento académico es la conciencia.

Presentamos en la Tabla 2 los resultados de las correlaciones entre el conjunto de factores estudiados y el rendimiento académico, ya desglosado en: calificaciones de exámenes (notas), asistencia a clase y dedicación al estudio. Si nos fijamos en las variables del rendimiento académico, las correlaciones significativas se agrupan en torno a la conciencia, bien como factor de personalidad, bien en las dos facetas utilizadas (CInd, CPlan).

El análisis de la correlación entre el factor de personalidad $\mathrm{C}$ y sus dos facetas analizadas, Trabajo continuo y Planificación, corrobora de la misma forma la relación entre ellas, obteniendo valores muy altos de $r=0.728$ para la faceta de Trabajo continuo y de $r=0.548$ para la faceta de Planificación.

\begin{tabular}{|c|c|c|c|c|c|c|c|c|}
\hline & & $\begin{array}{l}\text { E } \\
\text { Extra- } \\
\text { versión }\end{array}$ & $\begin{array}{l}\text { A } \\
\text { Amabili- } \\
\text { dad }\end{array}$ & $\begin{array}{l}\text { C } \\
\text { Concien- } \\
\text { cia }\end{array}$ & $\begin{array}{l}\mathbf{N} \\
\text { Neuroti } \\
\text {-cismo }\end{array}$ & $\begin{array}{l}\text { O } \\
\text { Aper- } \\
\text { tura } \\
\end{array}$ & $\begin{array}{l}\text { C } \\
\text { Ind }\end{array}$ & $\begin{array}{l}\text { C } \\
\text { Plan }\end{array}$ \\
\hline \multirow[t]{2}{*}{ NOTAS } & Pearson &,- 086 &,- 042 & ,416** &,- 004 & ,006 & ,403** & ,293** \\
\hline & Correlación &, 066 &, 372 &, 000 & ,936 & ,893 & ,000 & ,000 \\
\hline ASIST. & Pearson &,- 044 & ,074 & ,227** & ,039 &,- 031 & 320 ** & ,202** \\
\hline A CLASE & Correlación &, 346 &, 116 &, 000 &, 407 & ,509 &, 000 &, 000 \\
\hline DEDIC. & Pearson &,- 020 & ,031 & ,540** &,$- 094 *$ &,- 011 & ,733** & ,455** \\
\hline ESTUD. & Correlación & ,665 & ,509 & ,000 &, 044 &, 812 & ,000 & ,000 \\
\hline
\end{tabular}

Tabla 2. Correlaciones de Pearson entre los factores de personalidad y las dos facetas de la conciencia con los indicadores del rendimiento académico $(\mathrm{N}=456)$

A continuación se analizaron las diferencias entre géneros para todas las variables continuas. Los resultados están expuestos en la Tabla 3. 


\begin{tabular}{|c|c|c|c|c|c|}
\hline & Género & Media & DS & $t$ & $\begin{array}{l}\text { Diferencia } \\
\text { de medias }\end{array}$ \\
\hline \multirow{2}{*}{$\mathrm{E}$} & Hombres & 6,57 & 1,75 & \multirow{2}{*}{$-1,99$} & \multirow{2}{*}{,- 36} \\
\hline & Mujeres & 6,92 & 1,87 & & \\
\hline \multirow{2}{*}{ A } & Hombres & 5,53 & 1,69 & \multirow{2}{*}{,$- 49 *$} & \multirow{2}{*}{,- 08} \\
\hline & Mujeres & 5,61 & 1,71 & & \\
\hline \multirow{2}{*}{$\mathrm{C}$} & Hombres & 5,58 & 1,80 & \multirow{2}{*}{$-6,75$} & \multirow{2}{*}{$-1,20$} \\
\hline & Mujeres & 6,77 & 1,82 & & \\
\hline \multirow{2}{*}{$\mathrm{N}$} & Hombres & 6,36 & 1,96 & \multirow{2}{*}{4,84} & \multirow{2}{*}{,92 } \\
\hline & Mujeres & 5,43 & 1,95 & & \\
\hline \multirow{2}{*}{$\mathrm{O}$} & Hombres & 6,18 & 1,89 & \multirow{2}{*}{$1,07^{*}$} & \multirow{2}{*}{,20 } \\
\hline & Mujeres & 5,98 & 2,00 & & \\
\hline \multirow{2}{*}{ Cplan } & Hombres & 27,70 & 5,92 & \multirow{2}{*}{$-4,09$} & \multirow{2}{*}{$-2,40$} \\
\hline & Mujeres & 30,10 & 6,04 & & \\
\hline \multirow{2}{*}{ Cind } & Hombres & 29,32 & 6,93 & \multirow{2}{*}{$-6,82$} & \multirow{2}{*}{$-4,65$} \\
\hline & Mujeres & 33,97 & 7,02 & & \\
\hline \multirow{2}{*}{ Notas } & Hombres & 63,61 & 12,11 & \multirow{2}{*}{$-3,99$} & \multirow{2}{*}{$-4,43$} \\
\hline & Mujeres & 68,04 & 10,00 & & \\
\hline \multirow{2}{*}{ Asis } & Hombres & 87,30 & 15,40 & \multirow{2}{*}{$1,34^{*}$} & \multirow{2}{*}{2,01} \\
\hline & Mujeres & 85,29 & 15,43 & & \\
\hline \multirow{2}{*}{ Ded } & Hombres & 51,94 & 20,29 & \multirow{2}{*}{$-5,58$} & \multirow{2}{*}{$-10,26$} \\
\hline & Mujeres & 62,20 & 18,02 & & \\
\hline
\end{tabular}

Tabla 3. Prueba $t$ de Student de comparación de medias entre géneros para datos independientes de todas las variables continuas: E extraversión, A amabilidad, C conciencia, $\mathrm{N}$ estabilidad emocional, $\mathrm{O}$ apertura mental, Cplan conciencia planificación, Cind trabajo continuo, notas, asistencia a clase, y dedicación al estudio $(n$ hombres $=165 ; n$ mujeres $=291)$. $*$ dif. significativas para $p<.05$

Como se puede observar existen diferencias entre géneros en las variables continuas excepto en tres de ellas: amabilidad, apertura mental y asistencia a clase. El sentido de las diferencias indica que los hombres puntúan más alto en estabilidad emocional mientras que las mujeres obtienen valores más elevados en las variables de extraversión, conciencia, conciencia de planificación, conciencia de trabajo continuo, notas y dedicación al estudio.

\begin{tabular}{lccccc}
\hline & Grupo & Percentil & C & Cplan & Cind \\
\hline \multirow{2}{*}{$\begin{array}{l}\text { Hombre } \\
n=165\end{array}$} & Bajo & 25 & $\leq 4$ & $\leq 23$ & $\leq 24$ \\
\cline { 2 - 6 } & Medio & 50 & $5-6$ & $24-31$ & $25-34$ \\
\cline { 2 - 6 } & Alto & 75 & $\geq 7$ & $\geq 32$ & $\geq 35$ \\
\hline \multirow{2}{*}{$\begin{array}{l}\text { Mujer } \\
n=291\end{array}$} & Bajo & 25 & $\leq 5$ & $\leq 26$ & $\leq 29$ \\
\cline { 2 - 6 } & Medio & 50 & $6-7$ & $27-33$ & $26-38$ \\
\cline { 2 - 6 } & Alto & 75 & $\geq 8$ & $\geq 34$ & $\geq 39$ \\
\hline
\end{tabular}

Tabla 4. Grupos según la asignación de puntuaciones de $\mathrm{C}$ conciencia, Cplan conciencia planificación y Cind conciencia persistencia a los percentiles 25,50 y 75, de acuerdo al género del alumno $(n=456)$. 
Dado que las diferencias entre géneros están comprendidas en el principal objetivo del presente trabajo, que es la influencia de las variables de la conciencia en los indicadores del aprovechamiento académico, los análisis ANOVA que se han llevado a cabo a continuación se han realizado de forma separada para los hombres y las mujeres de la muestra. Para completar dichos ANOVA se han transformado las variables de la conciencia de continuas a categóricas en función de los tres percentiles 25,50 y 75 , que han dado lugar a tres grupos en cada variable, que denominaremos, respectivamente, $\mathrm{C}$ baja, $\mathrm{C}$ media, $\mathrm{C}$ alta, Cplan baja, Cplan media, Cplan alta, Cind baja, Cind media, Cind alta. Los valores de estas tres variables y su correspondencia con cada uno de los percentiles están expuestos en la Tabla 4.

Los resultados de los ANOVA calculados en base a los valores marcados por los percentiles de la Tabla 4 están expuestos en las Tablas 5 y 6 para hombres y mujeres, respectivamente.

\begin{tabular}{|c|c|c|c|c|c|}
\hline $\begin{array}{c}\text { Rendimiento } \\
\text { académico }\end{array}$ & $n$ & Media (DS) & $\begin{array}{l}\text { Sig. de } \\
\text { Levene }\end{array}$ & $\begin{array}{c}F \\
(2,164)\end{array}$ & $p$ \\
\hline \multirow{3}{*}{ Notas } & $\mathrm{C}$ baja $=47$ & $5,70(, 94)^{*}$ & \multirow{3}{*}{,07 } & \multirow{3}{*}{14,08} & \multirow{3}{*}{, 00} \\
\hline & $\mathrm{C}$ media $=71$ & $6,42(1,26)$ & & & \\
\hline & $\mathrm{C}$ alta $=47$ & $6,93(1,07)$ & & & \\
\hline \multirow{3}{*}{$\begin{array}{l}\text { Asistencia } \\
\text { a clase }\end{array}$} & $\mathrm{C}$ baja $=47$ & $83,83(15,40)^{*}$ & \multirow{3}{*}{,41 } & \multirow{3}{*}{2,84} & \multirow{3}{*}{, 06} \\
\hline & $\mathrm{C}$ media $=71$ & $86,97(16,38)$ & & & \\
\hline & $\mathrm{C}$ alta $=47$ & $91,28(13,12)^{*}$ & & & \\
\hline \multirow{3}{*}{$\begin{array}{l}\text { Dedicación } \\
\text { al estudio }\end{array}$} & $\mathrm{C}$ baja $=47$ & $39,79(18,24)^{*}$ & \multirow{3}{*}{,84 } & \multirow{3}{*}{21,30} & \multirow{3}{*}{, 00} \\
\hline & $\mathrm{C}$ media $=71$ & $51,83(18,31)^{*}$ & & & \\
\hline & $\mathrm{C}$ alta $=47$ & $64,26(17,91)^{*}$ & & & \\
\hline \multirow{3}{*}{ Notas } & Cplan baja $=42$ & $5,70(1,15)^{*}$ & \multirow{3}{*}{,99 } & \multirow{3}{*}{14,23} & \multirow{3}{*}{, 00} \\
\hline & Cplan media $=75$ & $6,34(1,12)^{*}$ & & & \\
\hline & Cplan alta $=48$ & $6,97(1,10)^{*}$ & & & \\
\hline \multirow{3}{*}{$\begin{array}{l}\text { Asistencia } \\
\text { a clase }\end{array}$} & Cplan baja $=42$ & $82,96(16,42)^{*}$ & \multirow{3}{*}{, 54} & \multirow{3}{*}{4,53} & \multirow{3}{*}{, 01} \\
\hline & Cplan media $=75$ & $86,60(15,49)$ & & & \\
\hline & Cplan alta $=48$ & $92,29(13,09)^{*}$ & & & \\
\hline \multirow{3}{*}{$\begin{array}{l}\text { Dedicación } \\
\text { al estudio }\end{array}$} & Cplan baja $=42$ & $38,57(19,93)^{*}$ & \multirow{3}{*}{,41 } & \multirow{3}{*}{15,80} & \multirow{3}{*}{, 00} \\
\hline & Cplan media $=75$ & $54,27(16,78)$ & & & \\
\hline & Cplan alta $=48$ & $60,00(20,42)$ & & & \\
\hline \multirow{3}{*}{ Notas } & Cind baja $=41$ & $5,67(, 95)^{*}$ & \multirow{3}{*}{ 11 } & \multirow{3}{*}{14,65} & \multirow{3}{*}{00} \\
\hline & Cind media $=80$ & $6,37(1,22)^{*}$ & & & \\
\hline & Cind alta $=44$ & $6,99(1,09)^{*}$ & & & \\
\hline
\end{tabular}

Tabla 5. ANOVA de los indicadores de aprovechamiento académico, notas, asistencia a clase y dedicación al estudio para tres grupos de las variables $\mathrm{C}$ conciencia, Cplan conciencia planificación y Cind conciencia persistencia. Submuestra de hombres $(n=165)$ 


\begin{tabular}{|c|c|c|c|c|c|}
\hline $\begin{array}{l}\text { Rendimiento } \\
\text { académico }\end{array}$ & $\mathrm{n}$ & Media (DS) & $\begin{array}{l}\text { Sig. de } \\
\text { Levene }\end{array}$ & $\begin{array}{c}\mathrm{F} \\
(2,164)\end{array}$ & $\mathrm{p}$ \\
\hline \multirow{3}{*}{$\begin{array}{l}\text { Asistencia } \\
\text { a clase }\end{array}$} & Cind baja $=41$ & $80,24(19,30)$ & \multirow{3}{*}{,06 } & \multirow{3}{*}{10,43} & \multirow{3}{*}{, 00} \\
\hline & Cind media $=80$ & $86,88(13,93)$ & & & \\
\hline & Cind alta $=44$ & $94,66(9,97)^{*}$ & & & \\
\hline \multirow{3}{*}{$\begin{array}{l}\text { Dedicación } \\
\text { al estudio }\end{array}$} & Cind baja $=41$ & $33,41(15,91)^{*}$ & \multirow{3}{*}{, 04} & \multirow{3}{*}{61,60} & \multirow{3}{*}{,00 } \\
\hline & Cind media $=80$ & $51,25(16,56)^{*}$ & & & \\
\hline & Cind alta $=44$ & $70,45(12,38)^{*}$ & & & \\
\hline
\end{tabular}

Tabla 5 (cont). ANOVA de los indicadores de aprovechamiento académico, notas, asistencia a clase y dedicación al estudio para tres grupos de las variables $\mathrm{C}$ conciencia, Cplan conciencia planificación y Cind conciencia persistencia. Submuestra de hombres $(n=165) *$ Subsets de Tukey significativamente distintos para $p<.05$

\begin{tabular}{|c|c|c|c|c|c|}
\hline $\begin{array}{l}\text { Rendimiento } \\
\text { académico }\end{array}$ & $n$ & Media (DS) & $\begin{array}{l}\text { Sig. de } \\
\text { Levene }\end{array}$ & $\begin{array}{c}F \\
(2,288) \\
\end{array}$ & $p$ \\
\hline \multirow{3}{*}{ Notas } & $\mathrm{C}$ baja $=84$ & $6,36(, 94)$ & \multirow{3}{*}{, 54} & \multirow{3}{*}{26,68} & \multirow{3}{*}{00} \\
\hline & $\mathrm{C}$ media $=94$ & $6,62(, 87)$ & & & \\
\hline & $\mathrm{C}$ alta $=113$ & $7,28(, 95)^{*}$ & & & \\
\hline \multirow{3}{*}{$\begin{array}{l}\text { Asistencia } \\
\text { a clase }\end{array}$} & $\mathrm{C}$ baja $=84$ & $78,59(16,56)^{*}$ & \multirow{3}{*}{, 02} & \multirow{3}{*}{12,28} & \multirow{3}{*}{00} \\
\hline & $\mathrm{C}$ media $=94$ & $86,70(14,25)$ & & & \\
\hline & $\mathrm{C}$ alta $=113$ & $89,03(14,01)$ & & & \\
\hline \multirow{3}{*}{$\begin{array}{l}\text { Dedicación } \\
\text { al estudio }\end{array}$} & $\mathrm{C}$ baja $=84$ & $49,29(17,06)^{*}$ & \multirow{3}{*}{,03 } & \multirow{3}{*}{49,70} & \multirow{3}{*}{, 00} \\
\hline & $\mathrm{C}$ media $=94$ & $62,34(15,69)^{*}$ & & & \\
\hline & $\mathrm{C}$ alta $=113$ & $71,68(14,32)^{*}$ & & & \\
\hline \multirow{3}{*}{ Notas } & Cplan baja $=79$ & $6,63(1,07)^{*}$ & \multirow{3}{*}{,45 } & \multirow{3}{*}{2,62} & \multirow{3}{*}{,08 } \\
\hline & Cplan media $=128$ & $6,79(, 95)$ & & & \\
\hline & Cplan alta $=84$ & $6,99(, 98)^{*}$ & & & \\
\hline \multirow{3}{*}{$\begin{array}{l}\text { Asistencia } \\
\text { a clase }\end{array}$} & Cplan baja $=79$ & $82,29(16,56)^{*}$ & \multirow{3}{*}{, 06} & \multirow{3}{*}{2,71} & \multirow{3}{*}{, 07} \\
\hline & Cplan media $=128$ & $85,47(15,10)$ & & & \\
\hline & Cplan alta $=84$ & $87,96(14,49)^{*}$ & & & \\
\hline \multirow{3}{*}{$\begin{array}{l}\text { Dedicación } \\
\text { al estudio }\end{array}$} & Cplan baja $=79$ & $51,90(18,33)^{*}$ & \multirow{3}{*}{, 13} & \multirow{3}{*}{23,00} & \multirow{3}{*}{00} \\
\hline & Cplan media $=128$ & $63,91(16,13)$ & & & \\
\hline & Cplan alta $=84$ & $69,29(16,27)$ & & & \\
\hline \multirow{3}{*}{ Notas } & Cind baja $=77$ & $6,34(, 89)^{*}$ & \multirow{3}{*}{,06 } & \multirow{3}{*}{16,24} & \multirow{3}{*}{00} \\
\hline & Cind media $=134$ & $6,84(, 91)^{*}$ & & & \\
\hline & Cind alta $=80$ & $7,20(1,08)^{*}$ & & & \\
\hline \multirow{3}{*}{$\begin{array}{l}\text { Asistencia } \\
\text { a clase }\end{array}$} & Cind baja $=77$ & $76,10(18,08)^{*}$ & \multirow{3}{*}{, 00} & \multirow{3}{*}{22,10} & \multirow{3}{*}{, 00} \\
\hline & Cind media $=134$ & $87,61(11,65)$ & & & \\
\hline & Cind alta $=80$ & $90,25(14,67)$ & & & \\
\hline & Cind baja $=77$ & $44,68(14,38)^{*}$ & & & \\
\hline Decicacion & Cind media $=134$ & $64,10(15,18)^{*}$ &, 00 & 102,17 & 00 \\
\hline & Cind alta $=80$ & $75,88(10,52)^{*}$ & & & \\
\hline
\end{tabular}

* Subsets de Tukey significativamente distintos para $p<.05$

Tabla 6. ANOVA de los indicadores de aprovechamiento académico, notas, asistencia a clase y dedicación al estudio para tres grupos de las variables $\mathrm{C}$ conciencia, Cplan conciencia planificación y Cind conciencia persistencia. Submuestra de mujeres $(n=291)$ 
Las Tablas 5 y 6 exponen los datos de los análisis de la varianza calculados para explorar el impacto de las puntuaciones en conciencia, planificación y trabajo continuo en los niveles de notas académicas, asistencia a clase y dedicación al estudio de los sujetos de la muestra. En dichas tablas se observa, en primer lugar, que la significación de los tests de Levene es mayoritariamente superior a .05, lo que indica que se puede asumir la igualdad de variancias entre los aspectos estudiados. Además, las series de ANOVAs que se han practicado para tres grupos de C, Cplan y Cind dan resultados en su mayoría significativos, tanto en el caso de los varones como en el de las mujeres. De estas series de análisis cabe destacar que los grupos de las tres variables utilizadas como independientes, C, Cplan y Cind, han demostrado ser capaces de predecir diferencias en las puntuaciones de los indicadores de aprovechamiento académico, principalmente en los referidos a la dedicación al estudio. La variable asistencia a clase, en cambio, no resulta predictible por las puntuaciones de la $\mathrm{C}$ en el caso de los alumnos de género masculino $(p=, 06)$, ni tampoco lo es a partir de las puntuaciones de la Cplan en el caso de las alumnas de la muestra $(p=, 07)$, aunque los resultados son francamente ajustados. Asimismo, para las estudiantes de género femenino las puntuaciones en Cplan tampoco resultan de interés a la hora de inferir sus notas académicas $(p=, 08)$.

\section{Discusión y conclusiones}

De los resultados obtenidos se desprenden algunas consideraciones valiosas de naturaleza psicoeducativa. Dichos resultados apuntan a una relación entre el rendimiento académico medido con tres variables distintas y el factor de personalidad de la conciencia. En este sentido coincidimos con las afirmaciones de autores como MacCann (2009) de que tanto la conciencia como sus facetas más elaboradas predicen en un alto grado el rendimiento académico y el futuro desarrollo en el comportamiento de la persona dentro del marco educacional. En el presente estudio se puede constatar como el factor $\mathrm{C}$ es el más significativo de entre todos los factores de personalidad estudiados. De este modo, los resultados concuerdan también con los estudios de autores como Beaujean et al., (2011), Duff, Boyle, Dunleavy y Ferguson, (2004), Johnson y Bloom, (1992), Komarraju, Karau, Schmeck y Avdic, (2011); MacCann, Duckworth y Roberts, (2012), Niño de Guzmán et al. (2003) y Poropat (2009).

Así pues, es posible corroborar que la variable de personalidad C, la conciencia, predice en función de su grado dentro del marco de la personalidad global de la persona, la capacidad que se posee para rendir en el contexto académico. Sin embargo, como hemos observado, no es la única variable a tener presente, puesto que la combinación de factores y de sus facetas puede servir de apoyo para inferir en otras variables que ayuden a mejorar el rendimiento. En el contexto de las facetas estudiadas, en concreto, la faceta de trabajo continuo se ajusta más a valores predictivos de éxito académico como ya apuntaron Dollinger, Matyja y Huber (2008) quienes tomaron la variable dedicación al estudio como muestra de la consecución de aprovechamiento académico. Con un mayor trabajo continuo y una mayor dedicación al estudio se favorecería la consecución de niveles más elevados de aprovechamiento académico. 
En nuestro estudio, al igual que señalan Schuman et al. (1985) también se observa que la asistencia a clase es menos relevante que la dedicación al estudio para predecir un buen rendimiento académico. Podemos indicar que los resultados muestran una relación entre niveles altos de conciencia con buena dedicación al estudio y un alto grado en la faceta de trabajo continuo. Estas tres variables nos llevan a afirmar que su correlación puede ser un buen predictor en el futuro para determinar posibles estudiantes con niveles de rendimiento académico alto o bajo. El rendimiento académico, es una variable que puede considerarse como algo dinámico y cambiante en el tiempo. Hasta la fecha, los niveles de rendimiento se valoraban sobre todo, a partir de las notas de los exámenes, siendo éstos, exámenes que en su mayoría potencian la memorización y la inteligencia lógico-matemática y la lingüística. Con la incorporación reciente de las nuevas teorías de inteligencia, y de nuevas didácticas escolares, la variable rendimiento está en proceso de cambio, aunque todavía siga siendo un indicador de logro alcanzado dentro de nuestro sistema educativo.

No obstante, cabe decir, que aunque el rendimiento académico sea una variable dinámica, sí es cierto que su relación con el Trabajo continuo, y en menor grado, también con la Planificación, ayudan al estudiante a obtener beneficio académico. La variable de personalidad $\mathrm{C}$, la conciencia, predice en función de su grado dentro del marco de la personalidad global de la persona, la capacidad más o menos que se tiene para rendir de forma académica. Sin embargo, como hemos observado, no es sólo la única variable a tener presente, puesto que sus facetas también puede servir de apoyo para inferir en otras variables que ayuden a mejorar el rendimiento, como pueden ser, a parte de las de personalidad, variables sociofamiliares y contextuales.

En cuanto a la variable género, de acuerdo a los resultados de MacCann et al. (2012), el género femenino tiene un nivel más alto de conciencia, sin embargo, los resultados no son significativos. En la misma dirección, los resultados de Carrasco y Del Barrio (2007), Del Barrio, Carrasco y Holgado (2006), MacCann et al. (2012), y Niño de Guzmán, et al. (2003), indican que el sexo femenino aumenta en niveles de conciencia. Según nuestros resultados se puede entrever que sí existen diferencias, tanto a nivel global de aprovechamiento académico respecto a la conciencia, como en cada una de las tres variables estudiadas. No es de extrañar, que variables que están ligadas al aprovechamiento académico y al factor $\mathrm{C}$ de personalidad sean consecuentes con los resultados en cuanto a diferencia de géneros. El aumento de conciencia en el género femenino se traduce asimismo en una mayor conciencia de trabajo continuo y de planificación. Esto a su vez, se refleja en los resultados de las variables utilizadas del rendimiento ya que el género femenino obtiene puntuaciones más elevadas en dedicación al estudio y en notas académicas. Los resultados globales no se ven afectados por la variable asistencia a clase, puesto que es la que menos incidencia tiene en el rendimiento académico así como en su relación con las facetas de la conciencia estudiadas.

En resumen, las variables de la conciencia analizadas en el presente trabajo, C, Cplan y Cind, son capaces de predecir niveles significativamente distintos de dedicación al estudio, de modo que los alumnos y alumnas que obtienen notas más altas en conciencia y sus dos facetas pasan una parte significativamente mayor de su 
tiempo libre aplicándose en las tareas de los estudios. Asimismo, las tres variables de la conciencia también son capaces de predecir valores distintos en las notas académicas y la asistencia a clase, en la misma dirección, de modo que los estudiantes que puntúan más alto en la conciencia y en las dos facetas de la misma obtienen notas más altas y asisten más a clase que sus iguales que puntúan más bajo en dicha dimensión de personalidad.

Cabría mencionar que la compleja tarea de aislar variables para el estudio del aprovechamiento académico, no está exenta de ser objeto de atención por parte de psicólogos y pedagogos. Fullana (1993) utiliza el criterio de "modificabilidad pedagógica" para referirse a la posibilidad de modificar cada variable de cada sujeto como resultado de una intervención educativa directa a través de recursos pedagógicos. De este modo, si se tiene en cuenta la variable de personalidad, conciencia, su planificación en el estudio y el trabajo continuo del alumno, uno de los recursos podría enfocarse a la tarea de crear hábitos de estudio y habilidades relacionadas con el mejoramiento de las facetas estudiadas. Y cómo apuntan Montero, Villalobos y Valverde (2007) el sistema educativo, y en concreto cada institución de enseñanza, debería poder ser responsable de realizar esfuerzos para implementar cambios que produzcan impactos deseados. De manera evidente este impacto sólo es posible en aquellas variables dependientes del sistema en sí mismo y las del propio alumno, como la creación de hábitos de estudio.

Somos conscientes, sin embargo, de que parte de las pruebas como son el autoinforme son subjetivas y cabe el problema de sesgo de deseabilidad social. Estas limitaciones pueden disminuir la consistencia en la medición de estas variables. También sería conveniente en futuras investigaciones ampliar el tamaño y edad de la muestra, y ver qué tipo de implicaciones tiene el modelo de personalidad de los cinco factores y su predictibilidad en otros ámbitos educativos y con la posibilidad de utilizar diferentes variables de aprovechamiento académico. No obstante, las diferencias individuales son un factor a tener presente en el futuro de las investigaciones dedicadas al rendimiento académico, así como el resto de facetas de la conciencia.

Debido al interés social que adquiere el ámbito educativo en relación al aprovechamiento académico, o en otras palabras, a la ausencia de fracaso escolar y bajo rendimiento, estudios similares pueden ofrecer pautas en la búsqueda de mejoras por parte de las instituciones educativas. Sabemos que el papel del educador, desde una perspectiva cualitativa, incide en el desarrollo de la personalidad, y la escuela puede aportar una mejor organización del tiempo que favorezca al alumno a una mayor constancia en los hábitos de estudio, aprender a planificarse y a aumentar la motivación de manera que redunde en una mayor dedicación al estudio y asistencia a clase. 


\section{Referencias bibliográficas}

ALLIK, J., y REALO, A. (1997). Intelligence, academic abilities, and personality. Personality and Individual Differences. 23, 809-814.

BEAUJEAN, A., FIRMIN, M., ATTAI, S., JOHNSON, C., FIRMIN, R., y MENA, K. (2011). Using personality and cognitive ability to predict academic achievement in a young adult sample. Personality and Individual Differences. 51, 709-714.

BUSATO, V., PRINS, F., ELSHOUT, J. y HAMAKER, C. (2000). Intellectual ability, learning style, achievement motivation and academic success of psychology students in higher education. Personality and Individual Differences. 29, 10571068.

CARRASCO, M.A., y DEL BARRIO, M.V. (2007). El modelo de los cinco grandes como predictor de la conducta agresiva en población infanto-juvenil. Revista de Psicopatología y Psicología clínica. 12,1. 23-32.

CHAMORRO-PREMUZIC, T., y FURNHAM, A. (2003). Personality predicts academic performance: Evidence from two longitudinal university samples. Journal of Research in Personality. 37, 319-338.

CLARIANA, M. (2008). Academic procrastination and class attendance. Are we really making it easier when attendance is not compulsory? En L. Gómez Chova, D. Martí Belenguer, e I. Candel Torres (Eds.), ICERI2008 Proceedings (pgs. 1-7). Valencia, Spain: International Association of Technology, Education and Development, IATED.

CONARD, M. (2006). Aptitude is not enough: How personality and behavior predict academic performance. Journal of Research in Personality. 40, 339-346.

COSTA, P.T., y McCRAE, R.R. (1992). NEO PI-R: Professional manual: Revised NEO PI-R and NEO-FFI. Florida: Psychological Assessment Resources, Inc.

DE LA FUENTE, J., PICHARDO, M., JUSTICIA, F., y BERBÉN, A. (2008). Enfoques de aprendizaje, autorregulación y rendimiento en tres universidades europeas. Psicothema. 20 (4), 705-711.

DE RAAD, B., y SHOUWENBURG, H (1996). Personality in learning and education. European Journal of Personality. 10, 303-336.

DEL BARRIO, M.V., CARRASCO, M.A., y HOLGADO, F.P. (2006). Análisis transversal de los cinco factores de personalidad por sexo y edad en niños españoles. Revista Latinoamericana de Psicología. 38, 3, 567-577.

DOLLINGER, S., MATYJA, A., y HUBER, J. (2008). Which factors best account for academic success: Those which college students can control or those they cannot? Journal of Research in Personality. 42, 872-885.

DUFF, A., BOYLE, E., DUNLEAVY, K., y FERGUSON, J. (2004). The relationship between personality, approach to learning and academic performance. Personality and Individual Differences. 36, 1907-1920. 
FULLANA, J. (1993). Revisió de la Recerca Educativa sobre les variables explicatives del Rendiment Acadèmic. Apunts per a l'ús del criteri de "modificabilitat pedagògica" de les variables. Estudi General. 185-200.

GARBANZO, G.M. (2007). Factores asociados al rendimiento académico en estudiantes universitarios, una reflexión desde la calidad de la educación superior pública. Revista Educación, 31 (1). 43-63.

JOHNSON, J., y BLOOM, M. (1994). An analysis of the contribution of the five factors of personality to variance in academic procrastination. Personality and Individual Differences. 18 (1), 127-133.

KOMARRAJU, M., KARAU, S., y SCHMECK, R. (2009). Role of the Big Five personality traits in predicting college students'academic motivation and achievement. Learning and Individual Differences. 19, 47-52.

KOMARRAJU, M., KARAU, S., SCHMECK, R., y AVDIC, A. (2011). The Big Five personality traits, learning styles, and academic achievement. Personality and Individual Differences. 51, 472-477.

KOMARRAJU, M., y KARAU, S. (2005). The relationship between the big five personality traits and academic motivation. Personality and Individual Differences. 39, 557-567.

LAIDRA, K., PULLMAN, H., y ALLIK, J. (2007). Personality and intelligence as predictors of academic achievement: A cross-sectional study from elementary to secondary school. Personality and Individual Differences, 42, 441-451.

LEÓN, B. (2008). Atención plena y rendimiento academic en estudiantes de enseñanza secundaria. European Journal of Education and Psychology. 3 (1), 17-26.

LOUNSBURY, J., SUNDSTROM, J., LOVELAND, J., y GIBSON, L. (2003). Intelligence, "Big Five" personality tratis, and work drive as predictors of course grade. Personality and Individual Differences. 35, 1231-1239.

MACCANN, C., DUCKWORTH, A., y ROBERTS, R. (2009). Empirical identification of the major facets of Conscientiousness. Learning and Individual Differences. 19, 451-458.

MACCANN, C., FOGARTY, G., y ROBERTS, R. (2012). Strategies for success in education: Time management is more important for part-time than full-time community college students. Learning and Individual Differences. 22, 618-623.

MONTERO, E., VILLALOBOS, J. y VALVERDE, A. (2007). Factores institucionales, pedagógicos, psicosociales y sociodemográficos asociados al rendimiento académico en la Universidad de Costa Rica.: Un análisis multinivel. Relieve, 13,2, 215-234.

NIÑO de GUZMÁN, I., CALDERÓN, A. y CASSARETTO, M. (2003). Personalidad y rendimiento academic en estudiantes universitarios. Revista de Psicología de PUCP.VOL. XXI, 1. 
NOFTLE, E., y ROBINS, R. (2007). Personality predictors of academic outcomes: Bif Five correlates of GPA and SAT scores. Journal of personality and Social Psychology. 93, 116-130.

O'CONNOR, M., y PAUNONEN, S. (2007). Big Five personality predictors of postsecondary academic performance. Personality and Individual Differences. 43, 971-990.

PEABODY, S., y DE RAAD, B. (2002). The substantive nature of psycholexical personality factors: A comparison across languages. Journal of personality and Social Psychology. 83, 983-997.

PERUGINI, M. y GALLUCCI, M. (1997). A hierarchical faceted model of the Big Five. European Journal of Personality. 11, 279-301.

POROPAT, A. (2009). A meta-analysis of the five-factor model of personality and academic performance. Psychollogical Bulletin. 135, 322-338.

RAMMSTEDT, B., y JOHN, O. P. (2007). Measuring personality in one minute or less: A 10-item short version of the Big Five Inventory in English and German. Journal of Research in Personality, 41, 203-212.

ROSE, R., HALL, J., BOLEN, C., y WEBSTER, R. (1996). Locus of control and college students' approaches to learning. Psychological Reports. 79, 163-171.

SAUCIER, G. y OSTENDORF, F. (1999). Hierarchical subcomponents of the Big Five personality factors: A cross-language replication. Journal of personality and Social Psychology. 76, 613-627.

SCHUMAN, H, WALSCH, E., OLSON, C., y ETHERIDGE, B. (1985). Effort and reward: The assumption that college grades are affected by quantity of study. Social Forces, 63, 945-966.

STERNBERG, R. y KAUFMAN, J. (1998). Human abilities. Annual Review of Psychology. 49, 479-502.

STIPEK, D. (2002). Motivation to learn: Integrating theory and practice. Boston, MA: Allyn \& Bacon.

WAGERMAN, S., y FUNDER, D. (2007). Acquaintance reports of personality and academic achievement: A case for conscientiousness. Journal of Research in Personality. 41, 221-229.

ZIEGLER, M., KNOGLER, M. y BÜHNER, M. (2009). Conscientiousness, achievement striving, and intelligence as performance predictors in a simple of German psychology students: Always a linear relationship? Learning and Individual Differences. 19, 288-292. 


\section{Correspondencia con los autores}

Teresa DEZCALLAR SÁEZ

Departamento de Psicología Básica, Evolutiva y de la Educación.

Facultad de Psicología, Universidad Autónoma de Barcelona.

Facultad de Psicología. Edificio B. Campus de Bellaterra 08193.

Cerdanyola del Vallès. Barcelona.

e-mail: Teresa.Dezcallar@uab.cat

Teléfono: 935814531 - 639123867 